\title{
On multidimensional generalized Cramér-Rao inequalities, uncertainty relations and characterizations of generalized $q$-Gaussian distributions
}

\author{
J F Bercher \\ Laboratoire d'informatique Gaspard Monge, UMR 8049 ESIEE-Paris, Université Paris-Est, \\ Paris, France \\ E-mail: jean-francois.bercher@univ-paris-est.fr
}

Received 13 September 2012, in final form 21 January 2013

Published 15 February 2013

Online at stacks.iop.org/JPhysA/46/095303

\begin{abstract}
In the present work, we show how the generalized Cramér-Rao inequality for the estimation of a parameter, presented in a recent paper, can be extended to the multidimensional case with general norms on $\mathbb{R}^{n}$, and to a wider context. As a particular case, we obtain a new multidimensional Cramér-Rao inequality which is saturated by generalized $q$-Gaussian distributions. We also give another related Cramér-Rao inequality, for a general norm, which is saturated as well by these distributions. Finally, we derive uncertainty relations from these Cramér-Rao inequalities. These uncertainty relations involve moments computed with respect to escort distributions, and we show that some of these relations are saturated by generalized $q$-Gaussian distributions. These results introduce extended versions of Fisher information, new Cramér-Rao inequalities, and new characterizations of generalized $q$-Gaussian distributions which are important in several areas of physics and mathematics.
\end{abstract}

PACS numbers: 02.50. $-\mathrm{r}, 05.90 .+\mathrm{m}, 89.70 .-\mathrm{a}$

Mathematics Subject Classification: 28D20, 94A17, 62B10, 39B62

\section{Introduction}

It is well known that the Gaussian distribution is a central distribution with respect to classical information measures and inequalities. In particular, the Gaussian distribution is both a maximum entropy and a minimum Fisher information distribution over all distributions with the same variance. We will show that the same kind of result holds for the family of generalized $q$-Gaussians, for Rényi or Tsallis entropy and a suitable extension of the Fisher information.

These generalized $q$-Gaussians appear in statistical physics, where they are the maximum entropy distributions of the nonextensive thermostatistics [1]. The generalized $q$-Gaussian 
distributions define a versatile family that can describe problems with compact support as well as problems with heavy tailed distributions. They are also analytical solutions of actual physical problems, see [2-5], and are sometimes known as Barenblatt-Pattle functions, following their identification by $[6,7]$. We shall also mention that the generalized $q$-Gaussian distributions appear in other fields, namely as the solution of nonlinear diffusion equations, or as the distributions that saturate some sharp inequalities in functional analysis [8-11].

In the literature, and in particular within nonextensive thermostatistics, several extensions of the Fisher information and of the Cramér-Rao inequality have been proposed, e.g. [12-16]. In information theory, the remarkable work by Lutwak et al $[17,18]$ also defines an extended Fisher information and a Cramér-Rao inequality saturated by $q$-Gaussian distributions. However, the Fisher information is originally defined in a broader context as the information about a parameter of a parametric family of distributions. It is only in the special case of a location parameter that it reduces to the Fisher information of the distribution. The Fisher information is especially important for the formulation of the Cramér-Rao inequality. This well-known inequality appears in the context of estimation theory, where it defines a lower bound on the variance of any estimator of a parameter.

In our recent work [19], we have thrown a bridge between concepts in estimation theory and tools of nonextensive thermostatistics. Using the notion of the escort distribution, we have established an extended version of the Cramér-Rao inequality for the estimation of a parameter. This new Cramér-Rao inequality includes the standard one, as well as Barankin-Vajda versions [20, corollary 5.1, 21] as particular cases. Furthermore, in the case of a location parameter, we have obtained extended versions of the standard Cramér-Rao inequality, which are saturated by the generalized $q$-Gaussians. This means that among all distributions with a given moment, the generalized $q$-Gaussians are also the minimizers of extended versions of the Fisher information, just as the standard Gaussian minimizes Fisher information over all distributions with a given variance. This result yields a new information-theoretic characterization of these generalized Gaussian distributions.

However, a quite frustrating point is that these results were limited to the univariate case, while the multidimensional case is obviously of high importance. This restriction is overcome in this paper where we show that previous results can be extended to the multidimensional case. More than that, we consider an even wider context where moments of the error are computed with respect to two different probability distributions. In addition, giving our results for general norms will be hardly more difficult than for Euclidean norms, so we consider this general case from the beginning. Finally, we derive new uncertainty relations from the multidimensional Cramér-Rao inequalities. Let us now give a brief overview of the results together with the organization of the paper.

Let $\theta \in \Theta \subseteq \mathbb{R}^{n}$ be a multidimensional parameter that we wish to estimate using data $x$. We show that for $\hat{\theta}(x)$ an estimator of $\theta$, if $f(x ; \theta)$ and $g(x ; \theta)$ are two probability densities, and if $\alpha$ and $\beta$ are Hölder conjugates of each other, then

$$
E\left[\|\hat{\theta}(x)-\theta\|^{\alpha}\right]^{\frac{1}{\alpha}} I_{\beta}[f \mid g ; \theta]^{\frac{1}{\beta}} \geqslant\left|n+\nabla_{\theta} \cdot B_{f}(\theta)\right|,
$$

where $\|$.$\| is a general norm on \mathbb{R}^{n}, \nabla_{\theta} . B_{f}(\theta)$ represents the divergence of the bias between $\hat{\theta}(x)$ and $\theta$, and $I_{\beta}[f \mid g ; \theta]$ stands for generalized Fisher information that measures the information in $f$ about $\theta$, and is taken with respect to $g$. This general result is established in section 3 . Then we discuss in subsection 3.2 some special cases of this general inequality. In particular, if $f$ and $g$ are a pair of $q$-escort distributions, then we obtain

$$
\begin{aligned}
& E\left[\|\hat{\theta}(x)-\theta\|^{\alpha}\right]^{\frac{1}{\alpha}} I_{\beta, q}[f \mid g ; \theta]^{\frac{1}{\beta}} \geqslant\left|n+\nabla_{\theta} \cdot E_{q}[\hat{\theta}(x)-\theta]\right| \\
& E_{\bar{q}}\left[\|\hat{\theta}(x)-\theta\|^{\alpha}\right]^{\frac{1}{\alpha}} I_{\beta, q}[f \mid g ; \theta]^{\frac{1}{\beta}} \geqslant\left|n+\nabla_{\theta} \cdot E[\hat{\theta}(x)-\theta]\right|,
\end{aligned}
$$


where $I_{\beta, q}[f \mid g ; \theta]$ is the generalized $(\beta, q)$-Fisher information and $E_{q}[$.$] denotes the$ $q$-expectation which is used in nonextensive statistics. These results are the multidimensional extensions, with an arbitrary norm, of our previous $q$-Cramér-Rao inequalities [19]. In the monodimensional case and $q=1$, these inequalities reduce to the Barankin-Vajda CramérRao inequality and to the standard Cramér-Rao inequality for $\alpha=\beta=2$. In addition, in the case of a location parameter, we show that

$$
E\left[\|x\|^{\alpha}\right]^{\frac{1}{\alpha}} I_{\beta, q}[g]^{\frac{1}{\beta}} \geqslant n
$$

which reduces again to our previous results in the univariate case. Examining carefully the cases for equality in (4), we exhibit that the lower bound is attained by generalized $q$-Gaussian distributions, and we prove that these generalized Gaussians are the unique extremal functions, provided that the dual norm is strictly convex. For a random vector $x$ in $\mathbb{R}^{n}$, these generalized $q$-Gaussians have the probability density

$$
G_{\gamma}(x)= \begin{cases}\frac{1}{Z(\gamma)}\left(1-(q-1) \gamma\|x\|^{\alpha}\right)_{+}^{\frac{1}{q-1}} & \text { for } q \neq 1 \\ \frac{1}{Z(\gamma)} \exp \left(-\gamma\|x\|^{\alpha}\right) & \text { if } q=1\end{cases}
$$

for $\alpha \in(0, \infty), \gamma$ a real positive parameter and $q>(n-\alpha) / n$, where we use the notation $(x)_{+}=\max \{x, 0\}$, and where $Z(\gamma)$ is the partition function ${ }^{1}$. For $q>1$, the density has a compact support, while for $q \leqslant 1$ it is defined on the whole $\mathbb{R}^{n}$ and behaves as a power distribution for $\|x\| \rightarrow \infty$. A shorthand notation for the expression of the generalized $q$-Gaussian density is

$$
G_{\gamma}(x)=\frac{1}{Z(\gamma)} \exp _{q^{*}}\left(-\gamma\|x\|^{\alpha}\right),
$$

with $q^{*}=2-q$ and where the so-called $q$-exponential function is defined by

$$
\exp _{q}(x):=(1+(1-q) x)_{+}^{\frac{1}{1-q}}, \quad \text { for } q \neq 1 \text { and } \exp _{q=1}(x):=\exp (x)
$$

In section 4 , we present another Cramér-Rao-type inequality which is also saturated by the generalized $q$-Gaussian. This inequality has been originally established by [17], and extended to the multidimensional case in [18] and independently in [22] in the case of an Euclidean norm. We show here that this last inequality can readily be stated and proved in the case of an arbitrary norm.

Finally, in section 5, we derive some new multidimensional uncertainty relations from the generalized $q$-Cramér-Rao inequalities. These uncertainty relations involve escort mean values and are saturated by the generalized Gaussians. In particular, we obtain an inequality of the form

$$
\left(E_{\frac{k}{2}}\left[\|x\|_{2}^{\gamma}\right]\right)^{\frac{1}{\gamma}}\left(E\left[\|\xi\|_{2}^{\theta}\right]\right)^{\frac{1}{\theta \lambda}}>\frac{1}{M_{\frac{k}{2}}\left[|\psi|^{2}\right]^{\frac{1}{k \lambda}}}\left(E_{\frac{k}{2}}\left[\|x\|_{2}^{\gamma}\right]\right)^{\frac{1}{\gamma}}\left(E\left[\|\xi\|_{2}^{\theta}\right]\right)^{\frac{1}{\theta \lambda}} \geqslant K,
$$

where $x$ and $\xi$ are two Fourier dual variables, $\gamma \geqslant 2, \theta \geqslant 2$ and $E_{\frac{k}{2}}$ [.] denotes an expectation computed with respect to an escort distribution of order $\frac{k}{2}$. The lower bound $K$ is fixed and, for $\gamma=\theta=2$, is attained when the underlying wavefunction is a $q$-Gaussian. For $q=1$,

1 In the case of the Euclidean norm, the general expressions of the main information measures attached to the generalized Gaussians are derived in appendix A of [22]. Similar expressions can readily be obtained in the case of a general norm, using the change of variable in polar coordinates $x=r u$, with $u=x /\|x\|$ and the representation of the Lebesgue measure $\mathrm{d} x=r^{n-1} \mathrm{~d} r \mathrm{~d} \sigma(u)$, cf [23, page 87], where $\mathrm{d} \sigma(u)$ denotes the surface element on the unit sphere. By this remark, the expressions in [22, appendix A] are valid, with the proviso that $\omega_{n}$ will denote the volume of the $n$-dimensional unit ball $\mathcal{B}=\left\{x \in \mathbb{R}^{n}:\|x\| \leqslant 1\right\}$. 
$\gamma=\theta=2$, (8) gives the multidimensional version of the well-known Weyl-Heisenberg uncertainty principle.

In order to derive these different inequalities, we will need some preliminary results, in particular concerning some properties of general norms on $\mathbb{R}^{n}$. This is the objective of section 2 where we first define the notion of dual norms and prove a result on the gradient of a general norm. Then, we establish, together with its equality conditions, a general Höldertype inequality. This inequality will be an essential ingredient in the derivation of the general Cramér-Rao inequality (1).

\section{Preliminary results}

As mentioned above, we will consider here general norms on $\mathbb{R}^{n}$. Let us first simply recall that a norm on $\mathbb{R}^{n}$ is a function $\|\|:. \mathbb{R}^{n} \rightarrow \mathbb{R}_{+}$such that for any $x, y \in \mathbb{R}^{n}$ and $\gamma \in \mathbb{R}$, then

(a) $\|\gamma x\|=|\gamma|\|x\|$, (b) $\|x+y\| \leqslant\|x\|+\|y\|, \quad$ and $\quad$ (c) $\|x\|=0$ iff $x=0$.

A large class of norms is the class of $L_{p}$-norms, $p \geqslant 1$, given by $\|x\|_{p}=\left(\sum_{i=1}^{n}|x|_{i}^{p}\right)^{\frac{1}{p}}$. As important particular cases, we have the $L_{1}$-norm, $\|x\|_{1}=\sum_{i=1}^{n}|x|_{i}$, the max-norm or $L_{\infty^{-}}$ norm $\|x\|_{\infty}=\max \left(\left|x_{1}\right|, \ldots,\left|x_{n}\right|\right)$ and of course the Euclidean $L_{2}$-norm $\|x\|_{2}=\left(\sum_{i=1}^{n} x_{i}^{2}\right)^{\frac{1}{2}}$. We shall mention that it is possible to use weighted versions of the norms above, e.g. $\|x\|_{w, p}=\left(\sum_{i=1}^{n} w_{i}|x|_{i}^{p}\right)^{\frac{1}{p}}$, with $w_{i}>0$, and that any injective linear transformation $A$ leads to a new norm, such as $\|x\|_{A}=\|A x\|$. Finally, it is also possible to construct new norms by combining different norms defined on subvectors of $x$.

A related important notion is the notion of dual norm. Let $E=\left(\mathbb{R}^{n},\|\cdot\|\right)$ be an $n$ dimensional normed space, where $\|$.$\| is an arbitrary norm, and let us denote E^{*}=\left(\mathbb{R}^{n},\|\cdot\|_{*}\right)$ its dual space. For $Y \in E^{*}$, the dual norm $\|\cdot\|_{*}$ is defined by

$$
\|Y\|_{*}=\sup _{\|X\| \leqslant 1} X . Y
$$

where $X . Y$ is the standard scalar product $X . Y=\sum_{i=1}^{n} X_{i} Y_{i}$. In particular, it is well known that if $\|$.$\| is an L_{p}$-norm, then $\|.\|_{*}$ is the $L_{q}$-norm, where $p$ and $q$ are the Hölder conjugates of each other, i.e. $p^{-1}+q^{-1}=1$, see e.g. [24, chapter 5]. By a direct consequence of the definition of the dual norm, we always have

$$
X . Y \leqslant\|X\|\|Y\|_{*} .
$$

Note that when the dot product $X . Y$ is negative, we can always take the minus of one of the elements to obtain $|X . Y|=X .(-Y) \leqslant\|X\|\|-Y\|_{*}=\|X\|\|Y\|_{*}$. Hence, we see that we actually have an extension of Hölder's inequality for vectors

$$
|X . Y| \leqslant\|X\|\|Y\|_{*} .
$$

Obviously, we recover here the Cauchy-Schwarz inequality if $\|\cdot\|=\|\cdot\|_{2}$ and the standard Hölder inequality for vectors if $\|\cdot\|=\|\cdot\|_{p}$, and thus $\|\cdot\|_{*}=\|\cdot\|_{q}$.

In the following, we will need several facts on the gradient of a norm. These facts are stated in the next lemma.

Lemma 1. Let $\|$.$\| be differentiable at x \in E$ and denote $x^{*}=\nabla_{x}\|\|.(x) \in E^{*}$ the gradient of the norm at $x$. The gradient of $\|x\|$ satisfies (a) $x . x^{*}=\|x\|$ and $(b)\left\|x^{*}\right\|_{*}=1$. Furthermore, when the dual norm $\|.\|_{*}$ is strictly convex, then the gradient $x^{*}$ is the unique vector that satisfies (a) and (b). 
Proof. We begin by equality (a). Let $x$ and $v$ be two vectors of $E$ and $\lambda$ be a real parameter. By the triangle inequality, we have $\|x+\lambda v\| \leqslant\|x\|+\lambda\|v\|$, so that

$$
\lim _{\lambda \rightarrow 0} \frac{\|x+\lambda v\|-\|x\|}{\lambda} \leqslant\|v\| \text {. }
$$

On the other hand, the chain rule for derivation $\frac{\mathrm{d}\|Z\|}{\mathrm{d} \lambda}=\frac{\mathrm{d} Z}{\mathrm{~d} \lambda} \cdot \nabla_{Z}\|Z\|$, with $Z=x+\lambda v$, gives

$$
\left.\frac{\mathrm{d}\|x+\lambda v\|}{\mathrm{d} \lambda}\right|_{\lambda=0}=v \cdot \nabla_{x}\|x\| \leqslant\|v\|,
$$

where the right inequality follows from (13). Of course, taking $v=x$ in (13) gives the equality sign, and (14) becomes $x . \nabla_{x}\|x\|=\|x\|$, that is (a).

By (14), we also obtain that

$$
\nabla_{x}\|x\| \cdot \frac{v}{\|v\|} \leqslant 1
$$

with equality, if $v=x$. Therefore, in the definition of the dual norm $\left\|\nabla_{x}\right\| x\|\|_{*}=$ $\sup _{\|w\| \leqslant 1} w \cdot \nabla_{x}\|x\|$, the supremum is equal to 1 and is attained for $w=x /\|x\|$. This proves (b).

In functional analysis, the existence of a solution to a system analogue to (a), (b) is granted by a consequence of the Hahn-Banach theorem, see e.g. the background material in [25]. In this context, the uniqueness of extension in the Hahn-Banach theorem, therefore the uniqueness of $x^{*}$, is guaranteed if the primal space $E$ is smooth, which in turn is equivalent to the strict convexity of the dual norm [26, chapter 2]. In our setting, it is easy to check that we have uniqueness of the solution to the system (a), (b) provided that the dual norm is strictly convex. Indeed, if $x_{1}^{*}$ and $x_{2}^{*}$ are two solutions to (a), (b), then we have by (b) $\frac{x}{\|x\|} \cdot\left(x_{1}^{*}+x_{2}^{*}\right)=2$. Accordingly, the dual norm $\left\|x_{1}^{*}+x_{2}^{*}\right\|_{*}=\sup _{\|w\| \leqslant 1} w \cdot\left(x_{1}^{*}+x_{2}^{*}\right)$ is necessarily greater than 2: $\left\|x_{1}^{*}+x_{2}^{*}\right\|_{*} \geqslant 2$. On the other hand, if the dual norm is strictly convex and using (a), we have $\left\|x_{1}^{*}+x_{2}^{*}\right\|_{*} \leqslant\left\|x_{1}^{*}\right\|_{*}+\left\|x_{2}^{*}\right\|_{*}=2$, with equality if and only if $x_{1}^{*}=x_{2}^{*}$. Combining the two inequalities, we see that the two solutions are necessarily equal. Finally, since we have already identified that the gradient of the norm satisfies (a), (b), we obtain the last item in the lemma.

The standard Hölder's inequality works for functions and relates the $L_{1}$ norm of the product of two functions to the product of their $L_{p}$ and $L_{q}$ norms: $\|f g\|_{1} \leqslant\|f\|_{p}\|g\|_{q}$, with $1 \leqslant p, q \leqslant \infty$ and $1 / p+1 / q=1$. For vectors and an arbitrary norm $\|$.$\| , inequality (12) gives$ another kind of Hölder inequality (actually, this inequality is also true in a broader context, see e.g. [27]). By combining these two inequalities, we obtain another Hölder-type inequality for vector-valued functions, which involves arbitrary norms. This inequality will be a key in the derivation of the new multidimensional Cramér-Rao inequality. It is given, with its equality condition, in the following lemma.

Lemma 2. Let $E=\left(\mathbb{R}^{n},\|\cdot\|\right)$ be an n-dimensional normed space and $E^{*}=\left(\mathbb{R}^{n},\|\cdot\|_{*}\right)$ be its dual space. If $X(t)$ and $Y(t)$ are two functions taking values respectively in $E$ and $E^{*}$, and if $w(t)$ is a weight function, then

$$
\begin{aligned}
\left(\int\|X(t)\|^{\alpha} w(t) \mathrm{d} t\right)^{\frac{1}{\alpha}}\left(\int\|Y(t)\|_{*}^{\beta} w(t) \mathrm{d} t\right)^{\frac{1}{\beta}} & \geqslant \int|X(t) . Y(t)| w(t) \mathrm{d} t, \\
& \geqslant\left|\int X(t) . Y(t) w(t) \mathrm{d} t\right|
\end{aligned}
$$

with $\alpha$ and $\beta$ being Hölder conjugates of each other, i.e. $\alpha^{-1}+\beta^{-1}=1, \alpha \geqslant 1$. The equality is obtained if

$$
Y(t)=K\|X(t)\|^{\alpha-1} \nabla_{X(t)}\|X(t)\|,
$$


with $K \in \mathbb{R}$ for inequality (16) and with $K \in \mathbb{R}_{+}$for the lower bound (17). If the dual norm is strictly convex, then the function $Y(t)$ in (18) above is the unique function which saturates inequalities (16)-(17).

Proof. By inequality (12), we have $|X(t) . Y(t)| \leqslant\|X(t)\|\|Y(t)\|_{*}$. Integrating this inequality with respect to $t$, we obtain

$$
\int|X(t) . Y(t)| w(t) \mathrm{d} t \leqslant \int\|X(t)\|\|Y(t)\|_{*} w(t) \mathrm{d} t .
$$

Obviously, we always have

$$
\left|\int X(t) . Y(t) w(t) \mathrm{d} t\right| \leqslant \int|X(t) . Y(t)| w(t) \mathrm{d} t
$$

with equality if $X(t) . Y(t) \geqslant 0$ everywhere. Then, it only remains to apply the standard Hölder inequality on the right-hand side of (19):

$$
\int\|X(t)\|\|Y(t)\|_{*} w(t) \mathrm{d} t \leqslant\left(\int\|X(t)\|^{\alpha} w(t) \mathrm{d} t\right)^{\frac{1}{\alpha}}\left(\int\|Y(t)\|_{*}^{\beta} w(t) \mathrm{d} t\right)^{\frac{1}{\beta}}
$$

to obtain (16). Inequality (17) is then followed by (20).

As far as the cases of equality are concerned, we know that in the Hölder inequality (21), the equality is obtained if and only if $\|Y(t)\|_{*}^{\beta}=K\|X(t)\|^{\alpha}$, with $K$ being a positive constant. Using the fact that $\alpha / \beta=\alpha-1$, the condition becomes $\|Y(t)\|_{*}=K\|X(t)\|^{\alpha-1}$. This condition implies that $Y(t)$ must be of the form

$$
Y(t)=K\|X(t)\|^{\alpha-1} u,
$$

where $u$ is a vector of $E^{*}$ with the unit norm: $\|u\|_{*}=1$. By inequality (12) we see that the integrand on the left-hand side of (19) is always less or equal to the integrand on the right. Therefore, we will only obtain equality in (19) if the integrands are equal. Then if we plug (22) in inequality (12), we obtain the condition $\|X(t)\|^{\alpha-1}|X(t) \cdot u|=\|X(t)\|^{\alpha}$, that is, finally $|X(t) \cdot u|=\|X(t)\|$. Since we know by lemma 1 that $v=\nabla_{X(t)}\|X(t)\|$ is a unit vector that satisfies $X(t) \cdot v=\|X(t)\|$, and is unique if the dual norm is strictly convex, we see that $u= \pm v= \pm \nabla_{X(t)}\|X(t)\|$ and this concludes the proof of the first inequality. For equality to hold in the lower bound (17), the integrand must be positive, which in turn implies that $X(t) . u=\|X(t)\|$ and $u=\nabla_{X(t)}\|X(t)\|$.

\section{The generalized Cramér-Rao inequality}

In this section, we first derive a main Cramér-Rao inequality for the estimation of a multidimensional parameter and introduce a generalized version of Fisher information. Next, we examine the particular case of a pair of escort distributions and then the case of a location parameter. Doing so, we obtain multidimensional versions of the $q$-Cramér-Rao inequality and a Cramér-Rao inequality characterizing generalized $q$-Gaussian distributions.

\subsection{The main Cramér-Rao inequality for the estimation of a parameter}

The problem of estimation is to determine a function $\hat{\theta}(x)$ in order to estimate an unknown parameter $\theta$. Let $f(x ; \theta)$ and $g(x ; \theta)$ be two probability density functions, with $x \in X \subseteq \mathbb{R}^{k}$ and $\theta$ be a parameter of these densities, $\theta \in \mathbb{R}^{n}$. An underlying idea in the statement of the new Cramér-Rao inequality is that it is possible to evaluate the moments of the error with respect to 
different probability distributions. For instance, in the estimation setting the estimation error is $\hat{\theta}(x)-\theta$. The bias can be evaluated with respect to $f$ according to

$$
B_{f}(\theta)=\int_{X}(\hat{\theta}(x)-\theta) f(x ; \theta) \mathrm{d} x=\mathrm{E}_{f}[\hat{\theta}(x)-\theta]
$$

while a general moment of a norm of the error can be computed with respect to another distribution, $g(x ; \theta)$, as in

$$
\mathrm{E}_{g}\left[\|\hat{\theta}(x)-\theta\|^{\beta}\right]=\int_{X}\|\hat{\theta}(x)-\theta\|^{\beta} g(x ; \theta) \mathrm{d} x .
$$

The distributions $f(x ; \theta)$ and $g(x, \theta)$ can be chosen arbitrarily and are not necessarily directly related. However, $g(x ; \theta)$ can be designed as a transformation of $f(x ; \theta)$ that highlights, or perhaps scores out, some characteristics of $f(x ; \theta)$. Typically, $g(x ; \theta)$ can be a weighted version of $f(x ; \theta)$, i.e. $g(x ; \theta)=h(x ; \theta) f(x ; \theta)$. The distribution $g(x ; \theta)$ can also be a quantized version of $f(x ; \theta)$, such as $g(x ; \theta)=[f(x ; \theta)]$, where [.] denotes the integer part. Another important special case is when $g(x ; \theta)$ is defined as the escort distribution of order $q$ of $f(x ; \theta)$, where $q$ plays the role of a tuning parameter. We will see that this special case, which is particularly important in the context of nonextensive statistical physics, will lead to generalized $q$-Gaussians as the extremal functions. We are now in a position to state and prove an extended version of the Cramér-Rao inequality.

Theorem 1. Let $f(x ; \theta)$ be a multivariate probability density function defined over a subset $X \subseteq \mathbb{R}^{n}$ and $\theta \in \Theta \subseteq \mathbb{R}^{k}$ be a parameter of the density. The set $\Theta$ is equipped with a norm $\|$.$\| , and the corresponding dual norm is denoted \|.\|_{*}$. Let $g(x ; \theta)$ denote another probability density function also defined on $(X ; \Theta)$. Assume that $f(x ; \theta)$ is a jointly measurable function of $x$ and $\theta$, is integrable with respect to $x$, is absolutely continuous with respect to $\theta$ and that the derivatives with respect to each component of $\theta$ are locally integrable. For any estimator $\hat{\theta}(x)$ of $\theta$, we have

$$
E_{g}\left[\|\hat{\theta}(x)-\theta\|^{\alpha}\right]^{\frac{1}{\alpha}} I_{\beta}[f \mid g ; \theta]^{\frac{1}{\beta}} \geqslant\left|n+\nabla_{\theta} \cdot B_{f}(\theta)\right|
$$

with $\alpha$ and $\beta$ being Hölder conjugates of each other, i.e. $\alpha^{-1}+\beta^{-1}=1, \alpha \geqslant 1$, and where the $(\beta, g)$-Fisher information

$$
I_{\beta}[f \mid g ; \theta]=\int_{X}\left\|\frac{\nabla_{\theta} f(x ; \theta)}{g(x ; \theta)}\right\|_{*}^{\beta} g(x ; \theta) \mathrm{d} x
$$

is the generalized Fisher information of order $\beta$ on the parameter $\theta$ contained in the distribution $f$ and taken with respect to $g$. The equality case is obtained if

$$
\frac{\nabla_{\theta} f(x ; \theta)}{g(x ; \theta)}=K\|\hat{\theta}(x)-\theta\|^{\alpha-1} \nabla_{\hat{\theta}(x)-\theta}\|\hat{\theta}(x)-\theta\|,
$$

with $K>0$.

Proof. The bias in (23) is an $n$-dimensional vector. Let us consider its divergence with respect to variations of $\theta$ :

$$
\operatorname{div} B_{f}(\theta)=\nabla_{\theta} \cdot B_{f}(\theta) .
$$

The regularity conditions in the statement of the theorem enable us to interchange integration with respect to $x$ and differentiation with respect to $\theta$, and

$\nabla_{\theta} \cdot B_{f}(\theta)=\int_{X} \nabla_{\theta} \cdot(\hat{\theta}(x)-\theta) f(x ; \theta) \mathrm{d} x+\int_{X} \nabla_{\theta} f(x ; \theta) .(\hat{\theta}(x)-\theta) \mathrm{d} x$. 
In the first term on the right, we have $\nabla_{\theta} \cdot \theta=n$, and the integral reduces to $-n \int_{X} f(x ; \theta) \mathrm{d} x=$ $-n$, since $f(x ; \theta)$ is a probability density on $X$. The second term can be rearranged so as to obtain an integration with respect to the density $g(x ; \theta)$, assuming that the derivatives with respect to each component of $\theta$ are absolutely continuous with respect to $g(x ; \theta)$, i.e. $g(x ; \theta) \gg \nabla_{\theta} f(x ; \theta)$. This gives

$$
n+\nabla_{\theta} \cdot B_{f}(\theta)=\int_{X} \frac{\nabla_{\theta} f(x ; \theta)}{g(x ; \theta)} \cdot(\hat{\theta}(x)-\theta) g(x ; \theta) \mathrm{d} x .
$$

Now, it only remains to apply the generalized Hölder-type inequality (17) in lemma 2 to the integral on the right side, with $X(x)=\hat{\theta}(x)-\theta, Y(x)=\frac{\nabla_{\theta} f(x ; \theta)}{g(x ; \theta)}$, and $w(x)=g(x ; \theta)$. This yields in all generality

$$
\left(\int_{X}\|\hat{\theta}(x)-\theta\|^{\alpha} g(x ; \theta) \mathrm{d} x\right)^{\frac{1}{\alpha}}\left(\int_{X}\left\|\frac{\nabla_{\theta} f(x ; \theta)}{g(x ; \theta)}\right\|_{*}^{\beta} g(x ; \theta) \mathrm{d} x\right)^{\frac{1}{\beta}} \geqslant\left|n+\nabla_{\theta} \cdot B_{f}(\theta)\right|
$$

which is (25). By lemma 2 again, we know that the case of equality occurs if $Y(t)=$ $K\|X(t)\|^{\alpha-1} \nabla_{X(t)}\|X(t)\|, K>0$, which gives (27).

\subsection{Main consequences of the general result}

3.2.1. Case of a q-escort distribution. Let $f(x ; \theta)$ and $g(x ; \theta)$ be a pair of $q$-escort distributions linked by

$$
f(x ; \theta)=\frac{g(x ; \theta)^{q}}{M_{q}[g ; \theta]} \quad \text { and } \quad g(x ; \theta)=\frac{f(x ; \theta)^{\bar{q}}}{M_{\bar{q}}[f ; \theta]},
$$

with $q>0, \bar{q}=1 / q$, and the information generating function $M_{q}[g ; \theta]$ defined by

$$
M_{q}[g ; \theta]=\int_{X} g(x ; \theta) \mathrm{d} x
$$

As usual, we will denote by $E_{q}$ [.] the $q$-expectation, which is the expectation taken with respect to an escort distribution of order $q$. Here we see that the expectation with respect to $f(x ; \theta)$ is also the $q$-expectation with respect to $g(x ; \theta)$. Let us also recall that the inverse function of the deformed $q$-exponential (7), the so-called $q$-logarithm, is defined by

$$
\ln _{q}(x):=\frac{x^{1-q}-1}{1-q} .
$$

With these notations, we have the following corollary of the general Cramér-Rao inequality.

Corollary 1. For the pair of escort distributions (32), the equivalent Cramér-Rao inequalities

$$
\begin{aligned}
& E\left[\|\hat{\theta}(x)-\theta\|^{\alpha}\right]^{\frac{1}{\alpha}} I_{\beta, q}[f \mid g ; \theta]^{\frac{1}{\beta}} \geqslant\left|n+\nabla_{\theta} \cdot E_{q}[\hat{\theta}(x)-\theta]\right| \\
& E_{\bar{q}}\left[\|\hat{\theta}(x)-\theta\|^{\alpha}\right]^{\frac{1}{\alpha}} I_{\beta, q}[f \mid g ; \theta]^{\frac{1}{\beta}} \geqslant\left|n+\nabla_{\theta} \cdot E[\hat{\theta}(x)-\theta]\right|,
\end{aligned}
$$

hold, where the generalized $(\beta, q)$-Fisher information is given by

and where the equality occurs if

$$
\begin{aligned}
I_{\beta, q}[f \mid g ; \theta] & =\frac{1}{M_{q}[g ; \theta]^{\beta}} E\left[g(x ; \theta)^{\beta(q-1)}\left\|\nabla_{\theta} \ln \frac{g(x ; \theta)^{q}}{M_{q}[g ; \theta]}\right\|_{*}^{\beta}\right] \\
& =M_{\bar{q}}[f ; \theta]^{\beta} E_{\bar{q}}\left[f(x ; \theta)^{\beta(1-\bar{q})}\left\|\nabla_{\theta} \ln f(x ; \theta)\right\|_{*}^{\beta}\right],
\end{aligned}
$$

$$
\nabla_{\theta} \ln _{\bar{q}} f(x ; \theta)=K\|\hat{\theta}(x)-\theta\|^{\alpha-1} \nabla_{\hat{\theta}(x)-\theta}\|\hat{\theta}(x)-\theta\|,
$$

with $K>0$. 
Proof. The Cramér-Rao inequalities (35) and (36) directly follow from the general CramérRao inequality (25), relations (32) between $f(x ; \theta)$ and $g(x ; \theta)$, and the notation of $q$-expectations. The expressions of the generalized $(\beta, q)$-Fisher information also follow by direct calculation. Finally, the equality condition yields

$$
f(x ; \theta)^{(1-\bar{q})} \nabla_{\theta} \ln f(x ; \theta)=K\|\hat{\theta}(x)-\theta\|^{\alpha-1} \nabla_{\hat{\theta}(x)-\theta}\|\hat{\theta}(x)-\theta\| .
$$

Noticing that the term on the left is nothing but the gradient of the deformed $q$-logarithm, $f(x ; \theta)^{(1-\bar{q})} \nabla_{\theta} \ln f(x ; \theta)=\nabla_{\theta} \ln _{\bar{q}} f(x ; \theta)$, we immediately obtain (39).

3.2.2. Case of a translation family. In the particular case of a translation parameter, the generalized Cramér-Rao inequality induces a new class of inequalities.

Let $\theta \in \mathbb{R}^{n}$ be a location parameter, $x \in X \subseteq \mathbb{R}^{n}$, and define the family of density $f(x ; \theta)=f(x-\theta)$ by $f(x ; \theta)$. In this case, we have $\nabla_{\theta} f(x ; \theta)=-\nabla_{x} f(x-\theta)$, provided that $f$ is differentiable at $x-\theta$, and the Fisher information becomes a characteristic of the information in the distribution. If $X$ is a bounded subset, then we will assume that $f(x)$ vanishes and is differentiable on the boundary $\partial X$. (Otherwise, the Fisher information defined for the function extended to $\mathbb{R}^{n}$ is not defined.)

Let us denote by $\mu_{f}$ the mean of $f(x)$. We immediately obtain that the mean of $f(x ; \theta)$ is $\left(\mu_{f}+\theta\right)$, so that an unbiased estimator of $\theta$ could be $\hat{\theta}(x)=x-\mu_{f}$. If we choose $\hat{\theta}(x)=x$, then the estimator will be biased, $B_{f}(\theta)=E_{q}[\hat{\theta}(x)-\theta]=\mu_{f}$, but independent of $\theta$, so that the gradient of the bias with respect to $\theta$ is zero. Under these conditions, the generalized Cramér-Rao inequality becomes

$$
\left(\int\|x-\theta\|^{\alpha} g(x ; \theta) \mathrm{d} x\right)^{\frac{1}{\alpha}}\left(\int\left\|\frac{\nabla_{x} f(x-\theta)}{g(x ; \theta)}\right\|_{*}^{\beta} g(x ; \theta) \mathrm{d} x\right)^{\frac{1}{\beta}} \geqslant n .
$$

Furthermore, we can also choose $\theta=0$, and obtain, as a corollary, the following interesting functional inequality.

Corollary 2. Let $f(x)$ and $g(x)$ be two multivariate probability density functions defined over a subset $X$ of $\mathbb{R}^{n}$. Assume that $f(x)$ is a measurable differentiable function of $x$, which vanishes and is differentiable on the boundary $\partial X$, that $\nabla_{x} f(x)$ is absolutely continuous with respect to $g(x)$ and finally that the involved integrals exist and are finite. Then, the following inequality holds:

$$
\left(\int_{X}\|x\|^{\alpha} g(x) \mathrm{d} x\right)^{\frac{1}{\alpha}}\left(\int_{X}\left\|\frac{\nabla_{x} f(x)}{g(x)}\right\|_{*}^{\beta} g(x) \mathrm{d} x\right)^{\frac{1}{\beta}} \geqslant n
$$

with equality if (and only if when the dual norm is strictly convex)

$$
\nabla_{x} f(x)=-K g(x)\|x\|^{\alpha-1} \nabla_{x}\|x\|,
$$

with $K>0$.

As an elementary application, let us consider the univariate case, with $X=$ $[0,1]$. Let us take for $g(x)$ the uniform distribution on the interval. Finally, let us choose for $f(x)$ a $\beta$-distribution: $f(x)=x^{a-1}(1-x)^{b-1} / B(a, b)$, with $B(a, b)$ being the $\beta$-function. Firstly, we obviously have $\int_{0}^{1}|x|^{\alpha} \mathrm{d} x=1$. Secondly, $f^{\prime}(x)=$ $\left((a-1) x^{a-2}(1-x)^{b-1}+(b-1) x^{a-1}(1-x)^{b-2}\right)$, so that the inequality is

$$
\left(\int_{0}^{1}\left|(a-1) x^{a-2}(1-x)^{b-1}+(b-1) x^{a-1}(1-x)^{b-2}\right|^{\beta} \mathrm{d} x\right)^{\frac{1}{\beta}} \geqslant B(a, b) \text {. }
$$

Taking now $\beta=1$ and $a>1, b>1$, we obtain the following inequality for $\beta$-functions:

$$
(a-1) B(a-1, b)+(b-1) B(a, b-1) \geqslant B(a, b) .
$$


3.2.3. Case of a location parameter within a pair of escort distributions. By combining the two aspects presented above, namely the case of a pair of escort distributions and the case of a location parameter, we will obtain a new Cramér-Rao inequality saturated by multivariate generalized $q$-Gaussians. This provides a new information theoretic characterization of generalized $q$-Gaussian and extends our previous results to the multivariate case and arbitrary norms. As in corollary 1 , we use a pair of $q$-escort distributions:

$$
f(x)=\frac{g(x)^{q}}{M_{q}[g]} \quad \text { and } \quad g(x)=\frac{f(x)^{\bar{q}}}{M_{\bar{q}}[f]},
$$

with $\bar{q}=1 / q$, and we denote by $E$ [.] the standard expectation with respect to $g(x)$, and by $E_{\bar{q}}$ [.] the $\bar{q}$-expectation with respect to $f(x)$, which is simply the standard expectation taken with respect to the escort $f(x)^{\bar{q}} / M_{\bar{q}}[f]$. In the statement of the following corollary, we will use the deformed exponential and logarithm defined in (7), (34). We will also use the notation $q_{*}=2-q$ that changes the quantities $\left(1-q_{*}\right)$ into $(q-1)$.

Corollary 3. Let $g(x)$ be a multivariate probability density function defined over a subset $X \subseteq \mathbb{R}^{n}$. Assume that $g(x)$ is a measurable differentiable function of $x$, which vanishes and is differentiable on the boundary $\partial X$, and finally that the involved integrals exist and are finite. Then, for the pair of escort distributions (46), the following q-Cramér-Rao inequality holds:

$$
m_{\alpha}[g]^{\frac{1}{\alpha}} I_{\beta, q}[g]^{\frac{1}{\beta}} \geqslant n
$$

with

$$
\left\{\begin{aligned}
m_{\alpha}[g] & =E\left[\|x\|^{\alpha}\right] \\
\bar{I}_{\beta, q}[g] & =\left(q / M_{q}[g]\right)^{\beta} E\left[g(x)^{\beta(q-1)}\left\|\nabla_{x} \ln g(x)\right\|_{*}^{\beta}\right], \\
& =\left(q / M_{q}[g]\right)^{\beta} E\left[\left\|\nabla_{x} \ln _{q *} g(x)\right\|_{*}^{\beta}\right]
\end{aligned}\right.
$$

where $\alpha$ and $\beta$ are Hölder conjugates of each other, i.e. $\alpha^{-1}+\beta^{-1}=1, \alpha \geqslant 1$, and where $I_{\beta, q}[g]$ denotes the generalized $(\beta, q)$-Fisher information.

In terms of $\bar{q}$-expectations with respect to $f(x)$, it can also be written as

$$
m_{\alpha, \bar{q}}[f]^{\frac{1}{\alpha}} \bar{I}_{\beta, q}[f]^{\frac{1}{\beta}} \geqslant n
$$

with

$$
\left\{\begin{aligned}
m_{\alpha, \bar{q}}[f] & =E_{\bar{q}}\left[\|x\|^{\alpha}\right] \\
\bar{I}_{\beta, q}[f] & =M_{\bar{q}}[f]^{\beta} E_{\bar{q}}\left[f(x)^{\beta(1-\bar{q})}\left\|\nabla_{x} \ln f(x)\right\|_{*}^{\beta}\right] . \\
& =M_{\bar{q}}[f]^{\beta} E_{\bar{q}}\left[\left\|\nabla_{x} \ln _{\bar{q}} f(x)\right\|_{*}^{\beta}\right]
\end{aligned}\right.
$$

In both cases, the equality occurs if

$f(x) \propto \exp _{\bar{q}}\left(-\gamma\|x\|^{\alpha}\right)$, or equivalently $g(x) \propto \exp _{q_{*}}\left(-\gamma\|x\|^{\alpha}\right)$, with $\gamma>0$.

If the dual norm is strictly convex, then this generalized q-Gaussian is the unique probability density function that achieves the equality in the extended Cramér-Rao inequalities.

Proof. As indicated above, the result is a direct consequence of theorem 1 in the case of a pair of escort distributions and of the estimation of a location parameter, with $\hat{\theta}(x)=x$ and $\theta=0$. Using (46), the condition for equality (43) becomes

$$
g(x)^{q-1} \nabla_{x} g(x)=-K\|x\|^{\alpha-1} \nabla_{x}\|x\| g(x) .
$$

From this equation, we see that $g(x)$ will only be a function of the norm of $x$, and therefore will be radially symmetric. Furthermore, we see that the gradient of $g(x)$ behaves as the negative 
of the gradient of $\|x\|$. This means that $g(x)$, which is a function of $\|x\|$, is non-increasing with $\|x\|$.

For $g(x) \neq 0$, the equality condition can be written as

$$
g(x)^{q-2} \nabla_{x} g(x)=\frac{1}{q-1} \nabla_{x} g(x)^{q-1}=-\frac{K}{\alpha} \nabla_{x}\|x\|^{\alpha},
$$

which, after integration of the two sides, gives

$$
g(x)^{q-1}=-\frac{K}{\alpha}(q-1)\|x\|^{\alpha}+C,
$$

where $C$ is a constant of integration. Since $g(x)$ is a probability density function, the solution is restricted to the domain where the right-hand side is non-negative, and $g(x)=0$ elsewhere. In particular, when $C$ is negative, we see that $g(x)$ vanishes around the origin and presents a singularity at $\|x\|=C \alpha / K(q-1)$. Since we assumed $g(x)$ differentiable everywhere, this solution must be discarded.

Therefore, the constant of integration $C$ must be positive, and

$$
g(x) \propto\left(1-\frac{K}{C \alpha}(q-1)\|x\|^{\alpha}\right)^{\frac{1}{q-1}} \propto \exp _{q_{*}}\left(-\gamma\|x\|^{\alpha}\right)
$$

which is (51). The expression of $f(x)$ simply follows from the fact that $f(x)$ is the escort distribution of $g(x)$.

\section{Another Cramér-Rao inequality saturated by generalized Gaussians}

We now turn to another Cramér-Rao-type inequality, which involves a variant $\phi_{\beta, q}[g]$ of the generalized Fisher information $I_{\beta, q}[g]$ above, and which is also saturated by generalized $q$-Gaussian distributions. However, this inequality is less directly related to estimation results than inequality (47) which is just a special case of the general Cramér-Rao inequality. The monodimensional version of this inequality has been established by [17] and extended to the multidimensional case in [18] and [22] in the case of a Euclidean norm. Actually, the inequality can readily be stated and proved for a general norm.

Theorem 2. For $n \geqslant 1, \beta$ and $\alpha$ Hölder conjugates of each other, $\alpha>1$, and $q>$ $\max \{(n-1) / n, n /(n+\alpha)\}$; then for any probability density $g$ on $\mathbb{R}^{n}$, supposed continuously differentiable and such that the involved information measures are finite,

$$
m_{\alpha}[g]^{\frac{1}{\alpha}} \phi_{\beta, q}[g]^{\frac{1}{\beta \lambda}} \geqslant m_{\alpha}[G]^{\frac{1}{\alpha}} \phi_{\beta, q}[G]^{\frac{1}{\beta \lambda}},
$$

with $\lambda=n(q-1)+1$ and where the general Fisher information is given by

$$
\phi_{\beta, q}[g]=\left(M_{q}[g] / q\right)^{\beta} I_{\beta, q}[g]=E\left[g(x)^{\beta(q-1)}\left\|\nabla_{x} \ln g(x)\right\|_{*}^{\beta}\right],
$$

and where the equality holds iff $g$ is a generalized Gaussian $g=G_{\gamma}$.

For the proof of this inequality, we will use two general inequalities relating the moment $m_{\alpha}[g]$, the generalized Fisher information $\phi_{\beta, q}[g]$ and the information generating function $M_{q}[g]=\int g(x)^{q} \mathrm{~d} x$. We will also use the notation $N_{q}[g]=M_{q}[g]^{\frac{1}{1-q}}$, which is sometimes known as the 'Rényi entropy power'. In the theorem above as well as in the lemma below, $\phi_{\beta, q}[G], m_{\alpha}[G]$ and $N_{q}[G]$ are the values taken by the Fisher information, the moment of order $\alpha$ and the entropy power when the probability density $g$ is the generalized $q$-Gaussian $G$. The exact expressions of these quantities are given in appendix A of [22], taking into account footnote 1 on page 3 . 
Lemma 3. For $n \geqslant 1, \alpha \in(0, \infty), q>n /(n+\alpha)$, and if $g$ is a probability density on random vectors of $\mathbb{R}^{n}$ with $m_{\alpha}[g]=E\left[\|x\|^{\alpha}\right]<\infty, N_{q}[g]<\infty$, then

$$
\frac{m_{\alpha}[g]^{\frac{1}{\alpha}}}{N_{q}[g]^{\frac{1}{n}}} \geqslant \frac{m_{\alpha}[G]^{\frac{1}{\alpha}}}{N_{q}[G]^{\frac{1}{n}}},
$$

with equality if and only if $g$ is a generalized Gaussian.

Proof. Inequality (58) has been stated and proved in [28] in the case of a Euclidean norm. We simply note here that the proof in [28] works as well in the case of a general norm.

We will also use a generalized Stam inequality derived from a general sharp GagliardoNirenberg inequality proved in the remarkable paper of Cordero et al [10].

Lemma 4. For $n \geqslant 1, \beta$ and $\alpha$ are the Hölder conjugates of each other, $\alpha>1$, and $q>\max \{(n-1) / n, n /(n+\alpha)\}$; then for any probability density on $\mathbb{R}^{n}$, supposed continuously differentiable, the following generalized Stam inequality holds:

$$
\phi_{\beta, q}[g]^{\frac{1}{\beta \lambda}} N_{q}[g]^{\frac{1}{n}} \geqslant \phi_{\beta, q}[G]^{\frac{1}{\beta \lambda}} N_{q}[G]^{\frac{1}{n}},
$$

with $\lambda=n(q-1)+1$ and with equality if and only if $g$ is any generalized $q$-Gaussian (6).

Proof. In our notations, the sharp Gagliardo-Nirenberg inequality [10, equation (34) p 320], with $a>1$ and $\|\nabla u\|_{\beta}=\left(\int\|\nabla u\|_{*}^{\beta} \mathrm{d} x\right)^{\frac{1}{\beta}}$, is

$$
\frac{\|\nabla u\|_{\beta}\|u\|_{a(\beta-1)+1}^{\frac{1}{\theta}-1}}{\|u\|_{a \beta}^{\frac{1}{\theta}}} \geqslant K,
$$

where $K$ is a sharp constant which is attained if and only if $u$ is a generalized Gaussian with exponent $1 /(1-a)$, and where $\theta$ is given by $\theta=n(a-1) / a(n \beta-(a \beta+1-a)(n-\beta))$. The idea is to take $u=g^{t}$, with $g$ being a probability density function, with $a \beta t=1$, and to note $q=[a(\beta-1)+1] t$. With these notations, we obtain that $\beta t=\beta(q-1)+1$, and (60) becomes

$$
\phi_{\beta, q}[g]^{\frac{1}{\beta}} N_{q}[g]^{\frac{(\theta-1)(1-q) t}{\theta q}} \geqslant K .
$$

Simplifying the expression of $\theta$ and the exponent in (61), we finally obtain, with $q<1$, the generalized Stam inequality (59), with equality if and only if $g$ is any generalized $q$-Gaussian (6). Actually, this generalized Stam inequality is also valid in the case $q>1$, as it can be checked from the results of [10] using similar steps as above. The conditions on $q$ simply ensure the existence of the information measures for the generalized Gaussian.

We end with the proof of theorem 2, which is now an easy task.

Proof. The Cramér-Rao inequality (47) can also be written as

$$
m_{\alpha}[g]^{\frac{1}{\alpha}} \frac{\phi_{\beta, q}[g]^{\frac{1}{\beta}}}{\frac{n}{q} M_{q}[g]} \geqslant 1 .
$$

Eliminating $M_{q}[g]$ between this inequality and the moment-entropy inequality (58) with $q>1$, we arrive at (56). Similarly, in the case $q<1$, the elimination of $M_{q}[g]$ between the extended $q$-Cramér-Rao inequality for a location parameter (62) and the generalized Stam inequality (59) also leads to (56). The case of equality directly follows from the cases of equality in the initial inequalities. Alternatively, we can observe that (56) also follows at once by the combination of (58) and (59). 


\section{Uncertainty relations associated with the $q$-Cramér-Rao inequalities}

It is known that the Weyl-Heisenberg uncertainty principle in statistical physics corresponds to the standard Cramér-Rao inequality for the location parameter, see e.g. [29]. Following this idea, we derive new uncertainty relations from the extended Cramér-Rao inequalities. These uncertainty relations involve escort mean values and are saturated by generalized Gaussians. These uncertainty relations thus give a way to measure the uncertainty not only with respect to the original wavefunction, but also with respect to an escort-deformed version of the associated probability density. Of course, the standard Weyl-Heisenberg uncertainty inequality is recovered in the case $q=1$.

Let us recall that the uncertainty principle originates in Heisenberg's work and is stated in Weyl's book [30, p 77], who credits Pauli. It indicates that if $x$ and $\xi$ are the dual Fourier variables (e.g. position and momentum), then the less the uncertainty in $x$, the greater in $\xi$ and conversely:

$$
E\left[|x|^{2}\right] E\left[|\xi|^{2}\right] \geqslant \frac{1}{16 \pi^{2}}
$$

with equality if and only if the probability density of $x$ is a Gaussian density. Many improvements and variations on this inequality have been given; see for instance the review [31]. Interesting, but not well-known, moment inequalities have been given in [32, 33]. Similar formulations are obtained below. Other entropic uncertainty relations have been stated by Hirshman [34] and improved in [35, 36]. It is worth mentioning that all the uncertainty inequalities are extremely useful in the analysis of complex systems, see the recent book [37], particularly the chapter [38], and the paper [39].

In the following, we consider a complex amplitude wavefunction $\psi(x), x \in \mathbb{R}^{n}$, with the unit Euclidean norm 2, and denote $\hat{\psi}(\xi)$ its Fourier transform, $\xi \in \mathbb{R}^{n}$. By the Parseval equality $\int_{\mathbb{R}^{n}}|\psi(x)|^{2} \mathrm{~d} x=\int_{\mathbb{R}^{n}}|\hat{\psi}(\xi)|^{2} \mathrm{~d} \xi=1$, and both $|\psi(x)|^{2}$ and $|\hat{\psi}(\xi)|^{2}$ are probability density functions.

We begin with a simple change of function which enables us to express the generalized Fisher information as a Dirichlet energy. This leads us to the following result.

Proposition 1. For $k=\beta /(\beta(q-1)+1), \lambda=n(q-1)+1$ and $q>$ $\max \{(n-1) / n, n /(n+\alpha)\}$, we have

$$
\begin{aligned}
& \frac{1}{M_{\frac{k q}{2}}\left[|\psi|^{2}\right]}\left(\int_{\mathbb{R}^{n}}\|x\|^{\alpha}|\psi(x)|^{k} \mathrm{~d} x\right)^{\frac{1}{\alpha}}\left(\int_{\mathbb{R}^{n}}\left\|\nabla_{x}|\psi(x)|\right\|_{*}^{\beta} \mathrm{d} x\right)^{\frac{1}{\beta}} \geqslant \frac{n}{k q} \\
& \frac{1}{M_{\frac{k}{2}}\left[|\psi|^{2}\right]^{\frac{1}{k \lambda}}}\left(E_{\frac{k}{2}}\left[\|x\|^{\alpha}\right]\right)^{\frac{1}{\alpha}}\left(\int_{\mathbb{R}^{n}}\left\|\nabla_{x}|\psi(x)|\right\|_{*}^{\beta} \mathrm{d} x\right)^{\frac{1}{\beta \lambda}} \geqslant \frac{1}{k^{\frac{1}{\lambda}}} m_{\alpha}[G]^{\frac{1}{\alpha}} \phi_{\beta, q}[G]^{\frac{1}{\beta \lambda}},
\end{aligned}
$$

where $E_{q}[$.$] denotes the q-expectation, the expectation computed with respect to the escort$ distribution of order $q$, and $M_{q}[$.$] the information generating function of order q$. In these inequalities, the case of equality is obtained if and only if $|\psi(x)|^{k} / M_{\frac{k}{2}}\left[|\psi|^{2}\right]$ is a generalized q-Gaussian.

Proof. Let

$$
f(x)=\frac{\left(|\psi(x)|^{2}\right)^{\frac{k}{2}}}{\int_{\mathbb{R}^{n}}\left(|\psi(x)|^{2}\right)^{\frac{k}{2}} \mathrm{~d} x}=\frac{|\psi(x)|^{k}}{M_{\frac{k}{2}}\left[|\psi|^{2}\right]},
$$


with $k=\beta /(\beta(q-1)+1)$. Note that we also have the conjugation relation $\frac{1}{\alpha}+\frac{1}{k}=q$. The change of function (66) is chosen in order to reduce $\phi_{\beta, q}[f]$ to the simple form (67) given below. On the other hand, the moment of order $\alpha$ with respect to $f(x)$ is nothing but the generalized $\frac{k}{2}$-escort moment (68) of order $\alpha$ computed with respect to $|\psi(x)|^{2}$. Finally, the information generating function $M_{q}[f]$ can also be expressed in terms of $|\psi(x)|^{2}$ :

$\phi_{\beta, q}[f]=\int_{\mathbb{R}^{n}} f(x)^{\beta(q-1)+1}\left\|\nabla_{x} \ln f(x)\right\|_{*}^{\beta} \mathrm{d} x=\frac{|k|^{\beta}}{M_{\frac{k}{2}}\left[|\psi|^{2}\right]^{\frac{\beta}{k}}} \int_{\mathbb{R}^{n}}\left\|\nabla_{x}|\psi(x, t)|\right\|_{*}^{\beta} \mathrm{d} x$.

$m_{\alpha}[f]=\int_{\mathbb{R}^{n}}\|x\|^{\alpha} \frac{\left(|\psi(x, t)|^{2}\right)^{\frac{k}{2}}}{\int_{\mathbb{R}^{n}}\left(|\psi(x, t)|^{2}\right)^{\frac{k}{2}} \mathrm{~d} x} \mathrm{~d} x=E_{\frac{k}{2}}\left[\|x\|^{\alpha}\right]$.

$M_{q}[f]=\frac{M_{\frac{k q}{2}}\left[|\psi|^{2}\right]}{M_{\frac{k}{2}}\left[|\psi|^{2}\right]^{q}}$

With these notations, the relation between $\phi_{\beta, q}[f]$ and $I_{\beta, q}[f]$, i.e. $I_{\beta, q}[f]^{\frac{1}{\beta}}=\frac{\phi_{\beta, q}[f]^{\frac{1}{\beta}}}{\frac{1}{q} M_{q}[f]}$, and the conjugation relation $\frac{1}{\alpha}+\frac{1}{k}=q$, the generalized Cramér-Rao (47), becomes (64). Similarly, the second Cramér-Rao inequality (56) gives (65).

Beginning with relations (64) and (65) and using a relation between the norm of a function and the norm of its Fourier transform, we obtain a pair of general uncertainty relations, for any exponent, that involve the expectations computed with respect to escort distributions. This result is stated as follows.

Theorem 3. For $2 \geqslant \alpha \geqslant 1, \beta$ its Hölder conjugate, $q>\max \{(n-1) / n, n /(n+\alpha)\}$, $k=\beta /(\beta(q-1)+1)$ and $\lambda=n(q-1)+1$; then we have

$$
\begin{aligned}
& \frac{M_{\frac{\alpha}{2}}\left[|\hat{\psi}|^{2}\right]^{\frac{1}{\alpha}} M_{\frac{k}{2}}\left[|\psi|^{2}\right]^{\frac{1}{\alpha}}}{M_{\frac{k q}{2}}\left[|\psi|^{2}\right]} E_{\frac{k}{2}}\left[\|x\|_{\alpha}^{\alpha}\right]^{\frac{1}{\alpha}} E_{\frac{\alpha}{2}}\left[\|\xi\|_{\beta}^{\beta}\right]^{\frac{1}{\beta}} \geqslant \frac{n}{2 \pi k q}\left(\frac{\beta^{\frac{1}{\beta}}}{\alpha^{\frac{1}{\alpha}}}\right)^{-\frac{n}{2 \beta}} \\
& \frac{M_{\frac{\alpha}{2}}\left[|\hat{\psi}|^{2}\right]^{\frac{1}{\alpha \lambda}}}{M_{\frac{k}{2}}\left[|\psi|^{2}\right]^{\frac{1}{k \lambda}}}\left(E_{\frac{k}{2}}\left[\|x\|_{\alpha}^{\alpha}\right]\right)^{\frac{1}{\alpha}}\left(E_{\frac{\alpha}{2}}\left[\|\xi\|_{\beta}^{\beta}\right]\right)^{\frac{1}{\beta \lambda}} \geqslant \frac{1}{(2 \pi k)^{\frac{1}{\lambda}}}\left(\frac{\beta^{\frac{1}{\beta}}}{\alpha^{\frac{1}{\alpha}}}\right)^{-\frac{n}{2 \beta \lambda}} m_{\alpha}[G]^{\frac{1}{\alpha}} \phi_{\beta, q}[G]^{\frac{1}{\beta \lambda}} .
\end{aligned}
$$

Proof. We first observe that for any complex-valued function, we have

$$
\left(\frac{\partial|\psi(x)|}{\partial x_{i}}\right)^{2}=\frac{\partial \psi(x)}{\partial x_{i}} \frac{\partial \psi(x)^{*}}{\partial x_{i}}-|\psi(x)|^{2}\left(\frac{\partial \arg \psi(x)}{\partial x_{i}}\right)^{2} .
$$

This relation implies that

$$
\left(\frac{\partial|\psi(x)|}{\partial x_{i}}\right) \leqslant\left|\frac{\partial \psi(x)}{\partial x_{i}}\right|
$$

with equality if and only if $\arg \psi(x)=c$, where $c$ is any constant. Therefore, we see that we always have $\left\|\nabla_{x}|\psi(x, t)|\right\|_{*} \leqslant\left\|\left|\nabla_{x} \psi(x, t)\right|\right\|_{*}$. In the following, we will take for $\|.\|_{*}$ a $\beta$-norm and for $\|$.$\| its dual \alpha$-norm. Doing so, we obtain $\left\|\left|\nabla_{x} \psi(x, t)\right|\right\|_{\beta}^{\beta}=\sum_{i=1}^{n}\left|\partial_{i} \psi(x)\right|^{\beta}$.

At this point, we can invoke the sharp version of the Hausdorff-Young inequality due to Babenko and Beckner [40], which states that for a pair of Fourier transforms $g$ and $\hat{g}$; then for $1 \leqslant \alpha \leqslant 2$,

$$
\|g\|_{\beta} \leqslant\left(\frac{\beta^{\frac{1}{\beta}}}{\alpha^{\frac{1}{\alpha}}}\right)^{\frac{n}{2}}\|\hat{g}\|_{\alpha},
$$


with equality if and only if $g$ is a Gaussian function or if $\alpha=\beta=2$ (Parseval's identity). Thus, it comes

$$
\int_{\mathbb{R}^{n}}\left\|\nabla_{x}|\psi(x, t)|\right\|_{\beta}^{\beta} \mathrm{d} x=\sum_{i=1}^{n} \int_{\mathbb{R}^{n}}\left|\partial_{i} \psi(x)\right|^{\beta} \mathrm{d} x \leqslant\left(\frac{\beta^{\frac{1}{\beta}}}{\alpha^{\frac{1}{\alpha}}}\right)^{\frac{n}{2}}(2 \pi)^{\beta} \sum_{i=1}^{n}\left(\int_{\mathbb{R}^{n}}\left|\xi_{i} \hat{\psi}(\xi)\right|^{\alpha} \mathrm{d} \xi\right)^{\frac{\beta}{\alpha}} .
$$

Inserting this inequality in (64), and taking account of the fact that $\|x\|^{\alpha}=\|x\|_{\alpha}^{\alpha}=\sum_{i=1}^{n}\left|x_{i}\right|^{\alpha}$, we obtain

$$
\frac{1}{M_{\frac{k q}{2}}\left[|\psi|^{2}\right]}\left(\sum_{i=1}^{n} \int_{\mathbb{R}^{n}}\left|x_{i}\right|^{\alpha}|\psi(x, t)|^{k} \mathrm{~d} x\right)^{\frac{1}{\alpha}}\left(\sum_{i=1}^{n}\left(\int_{\mathbb{R}^{n}}\left|\xi_{i}\right|^{\alpha}|\hat{\psi}(\xi)|^{\alpha} \mathrm{d} \xi\right)^{\frac{\beta}{\alpha}}\right)^{\frac{1}{\beta}} \geqslant \frac{n}{2 \pi k q}\left(\frac{\beta^{\frac{1}{\beta}}}{\alpha^{\frac{1}{\alpha}}}\right)^{-\frac{n}{2 \beta}} .
$$

Now, we can observe that by the Hölder inequality

$$
\left(\int_{\mathbb{R}^{n}}\left|\xi_{i}\right|^{\alpha}|\hat{\psi}(\xi)|^{\alpha} \mathrm{d} \xi\right) \leqslant\left(\int_{\mathbb{R}^{n}}\left|\xi_{i}\right|^{\beta}|\hat{\psi}(\xi)|^{\alpha} \mathrm{d} \xi\right)^{\frac{\alpha}{\beta}}\left(\int_{\mathbb{R}^{n}}|\hat{\psi}(\xi)|^{\alpha} \mathrm{d} \xi\right)^{1-\frac{\alpha}{\beta}} .
$$

Plugging this inequality in (76), we see that the exponent $\beta / \alpha$ simplifies, and that the inequality can be written in a weaker but more symmetric form:

$$
\frac{M_{\frac{\alpha}{2}}\left[|\hat{\psi}|^{2}\right]^{\frac{1}{\alpha}-\frac{1}{\beta}}}{M_{\frac{k q}{2}}\left[|\psi|^{2}\right]}\left(\int_{\mathbb{R}^{n}}\|x\|_{\alpha}^{\alpha}|\psi(x, t)|^{k} \mathrm{~d} x\right)^{\frac{1}{\alpha}}\left(\int_{\mathbb{R}^{n}}\|\xi\|_{\beta}^{\beta}|\hat{\psi}(\xi)|^{\alpha} \mathrm{d} \xi\right)^{\frac{1}{\beta}} \geqslant \frac{n}{2 \pi k q}\left(\frac{\beta^{\frac{1}{\beta}}}{\alpha^{\frac{1}{\alpha}}}\right)^{-\frac{n}{2 \beta}}
$$

which can also be written in terms of escort expectations as (70).

For the second Cramér-Rao inequality, we follow the very same steps, beginning with (65). By (75) it comes

$$
\begin{gathered}
\frac{1}{M_{\frac{k}{2}}\left[|\psi|^{2}\right]^{\frac{1}{k \lambda}}}\left(E_{\frac{k}{2}}\left[\|x\|_{\alpha}^{\alpha}\right]\right)^{\frac{1}{\alpha}}\left(\sum_{i=1}^{n}\left(\int_{\mathbb{R}^{n}}\left|\xi_{i} \hat{\psi}(\xi)\right|^{\alpha} \mathrm{d} \xi\right)^{\frac{\beta}{\alpha}}\right)^{\frac{1}{\beta \lambda}} \\
\geqslant \frac{1}{(2 \pi k)^{\frac{1}{\lambda}}} m_{\alpha}[G]^{\frac{1}{\alpha}} \phi_{\beta, q}[G]^{\frac{1}{\beta \lambda}}\left(\frac{\beta^{\frac{1}{\beta}}}{\alpha^{\frac{1}{\alpha}}}\right)^{-\frac{n}{2 \beta \lambda}},
\end{gathered}
$$

which, by the Hölder inequality (77), gives (71).

In the general case, inequalities (70) and (71) are not sharp, because they follow from the Babenko-Beckner inequality (74) and the Hölder inequality (77), where the conditions for equality are not met simultaneously. However, we can still get a sharp uncertainty relation, saturated by generalized $q$-Gaussians, in the case when $\alpha=\beta=2$. Of course, for $q=1$ (which, with $\beta=2$ gives $k=2$ ), we obtain a multidimensional version of Heisenberg inequality, which reduces to (63) in the scalar case.

Corollary 4. For $k=\beta /(\beta(q-1)+1), \lambda=n(q-1)+1, q>\max \{(n-1) / n, n /(n+\alpha)\}$ and $\gamma \geqslant 2, \theta \geqslant 2$, the following uncertainty relations hold:

$$
\begin{aligned}
& \frac{M_{\frac{k}{2}}\left[|\psi|^{2}\right]^{\frac{1}{2}}}{M_{\frac{k q}{2}}\left[|\psi|^{2}\right]} E_{\frac{k}{2}}\left[\|x\|_{2}^{\gamma}\right]^{\frac{1}{\gamma}} E\left[\|\xi\|_{2}^{\theta}\right]^{\frac{1}{\theta}} \geqslant \frac{n}{2 \pi k q}, \\
& \frac{1}{M_{\frac{k}{2}}\left[|\psi|^{2}\right]^{\frac{1}{k \lambda}}}\left(E_{\frac{k}{2}}\left[\|x\|_{2}^{\gamma}\right]\right)^{\frac{1}{\gamma}}\left(E\left[\|\xi\|_{2}^{\theta}\right]\right)^{\frac{1}{\theta \lambda}} \geqslant \frac{1}{(2 \pi k)^{\frac{1}{\lambda}}} m_{2}[G]^{\frac{1}{2}} \phi_{2, q}[G]^{\frac{1}{2 \lambda}} .
\end{aligned}
$$


For $\gamma=\theta=2$, the lower bound is attained if and only $|\psi(x)|^{k} / M_{\frac{k}{2}}\left[|\psi|^{2}\right]$ is a generalized $q$-Gaussian distribution, with $\arg \psi(x)=c$, where $c$ is a real constant. For $\frac{3}{2}-\frac{1}{\beta}>q$, we also have

$$
\left(E_{\frac{k}{2}}\left[\|x\|_{2}^{\gamma}\right]\right)^{\frac{1}{\gamma}}\left(E\left[\|\xi\|_{2}^{\theta}\right]\right)^{\frac{1}{\theta \lambda}} \geqslant \frac{1}{(2 \pi k)^{\frac{1}{\lambda}}} m_{2}[G]^{\frac{1}{2}} \phi_{2, q}[G]^{\frac{1}{2 \lambda}}
$$

Proof. Equality in (73) implies that $\arg \psi(x)=c$, where $c$ is a real constant. In the case $\alpha=\beta=2$, the Babenko-Beckner inequality reduces to Parseval's equality, and the Hölder inequality (77) is an identity. Therefore, we directly obtain

$$
\frac{1}{M_{\frac{k q}{2}}\left[|\psi|^{2}\right]}\left(\int_{\mathbb{R}^{n}}\|x\|_{2}^{2}|\psi(x, t)|^{k} \mathrm{~d} x\right)^{\frac{1}{2}}\left(\int_{\mathbb{R}^{n}}\|\xi\|_{2}^{2}|\hat{\psi}(\xi)|^{2} \mathrm{~d} \xi\right)^{\frac{1}{2}} \geqslant \frac{n}{2 \pi k q},
$$

which can also be written in terms of escort expectations as

$$
\frac{M_{\frac{k}{2}}\left[|\psi|^{2}\right]^{\frac{1}{2}}}{M_{\frac{k q}{2}}\left[|\psi|^{2}\right]} E_{\frac{k}{2}}\left[\|x\|_{2}^{2}\right]^{\frac{1}{2}} E\left[\|\xi\|_{2}^{2}\right]^{\frac{1}{2}} \geqslant \frac{n}{2 \pi k q} .
$$

Similarly, inequality (71) gives

$$
\frac{1}{M_{\frac{k}{2}}\left[|\psi|^{2}\right]^{\frac{1}{k \lambda}}}\left(E_{\frac{k}{2}}\left[\|x\|_{2}^{2}\right]\right)^{\frac{1}{2}}\left(E\left[\|\xi\|_{2}^{2}\right]\right)^{\frac{1}{2 \lambda}} \geqslant \frac{1}{(2 \pi k)^{\frac{1}{\lambda}}} m_{2}[G]^{\frac{1}{2}} \phi_{2, q}[G]^{\frac{1}{2 \lambda}} .
$$

In both inequalities, the lower bound is attained if $f(x)=|\psi(x)|^{k} / M_{\frac{k}{2}}\left[|\psi|^{2}\right]$ is a generalized $q$ Gaussian (which means that $|\psi(x)|^{2}$ is also a generalized $q$-Gaussian with a different entropic index: $\left.q^{\prime}=1+k(q-1) / 2\right)$.

By Jensen's inequality, we know that for $b \geqslant a$, we always have $E\left[|X|^{b}\right]^{\frac{a}{b}} \geqslant E\left[|X|^{a}\right]$. Therefore, applying this inequality with $\gamma \geqslant 2, \theta \geqslant 2$, we obtain (80) and (81). Finally, by the general power mean inequality, we know that $M_{a}[f]^{\frac{1}{a}} \geqslant M_{b}[f]^{\frac{1}{b}}$ for $a \geqslant b$. Therefore, when $k>2$, that is, $\frac{3}{2}-\frac{1}{\beta}>q$, then $M_{\frac{k}{2}}>M_{1}=1$, and inequality (81) yields (82).

\section{Conclusions}

This paper improves and builds on our previous findings presented in [19]. We connect concepts in estimation theory to tools used in nonextensive thermostatistics and establish general Cramér-Rao-type inequalities valid for estimation purposes. These results are given in the multidimensional case, and a feature of our approach is that it works for arbitrary norms on $\mathbb{R}^{n}$. As a direct consequence, we obtain multidimensional versions of our $q$-CramérRao inequalities, which include the Barankin-Vajda as well as the standard Cramér-Rao inequality as particular cases. Furthermore, in the case of a translation family, we have shown that the corresponding Cramér-Rao-type inequality is saturated by multidimensional $q$-Gaussian distributions. We have also presented a related general Cramér-Rao inequality which is saturated by the same $q$-Gaussian distributions. These results imply in particular that the generalized $q$-Gaussians are the minimizers of an extended version of the Fisher information, among all distributions with a given moment, just as the standard Gaussian minimizes Fisher information over all distributions with a given variance. Since these generalized Gaussians are already known to be the maximum entropy distributions for Rényi or Tsallis entropies, this yields a new, complementary, information theoretic characterization of these distributions. Finally, we have derived new multidimensional uncertainty relations from the extended Cramér-Rao inequalities. These uncertainty relations involve generalized 
expectations computed with respect to escort distributions, and we have shown that some of these uncertainty relations are saturated by generalized $q$-Gaussian distributions, thus generalizing the fact that the standard Heisenberg uncertainty relation is saturated by a standard Gaussian.

Some of the presented uncertainty inequalities are not sharp, and it would certainly be of interest to try to improve them, by looking to the extremal functions or to optimal constants. It would also be interesting to try to document the properties of the two generalized Fisher information. Finally, in their recent work [18], Lutwak et al have introduced an abstract, implicit, notion of Fisher information matrix attached to a probability density. It would be of interest to examine whether this notion could be extended and interpreted in the estimation theory framework.

\section{Acknowledgments}

The author thanks the anonymous referees for their suggestions that helped improve the article. The author is also very grateful to Deane Yang (Polytechnic Institute, NY), who suggested to look at general norms, and to Matthieu Fradelizi (LAMA, Université Paris-Est), for helpful discussions related to this work. Thanks are extended to Lodie Garbell for her friendly proofreading of the manuscript.

\section{References}

[1] Tsallis C 2009 Introduction to Nonextensive Statistical Mechanics (Berlin: Springer)

[2] Lutz E 2003 Anomalous diffusion and Tsallis statistics in an optical lattice Phys. Rev. A 67051402

[3] Schwämmle V, Nobre F D and Tsallis C 2008 q-Gaussians in the porous-medium equation: stability and time evolution Eur. Phys. J. B 66 537-46

[4] Vignat C and Plastino A 2009 Why is the detection of $q$-Gaussian behavior such a common occurrence? Physica A 388 601-8

[5] Ohara A and Wada T 2010 Information geometry of $q$-Gaussian densities and behaviors of solutions to related diffusion equations J. Phys. A: Math. Theor. 43035002

[6] Barenblatt G I 1952 On some unsteady motions of a liquid and a gas in a porous medium Prikl. Mat. Mech. 16 67-78

[7] Pattle R E 1959 Diffusion from an instantaneous point source with concentration dependent coefficient $Q$. J. Mech. Appl. Math. 12 407-9

[8] Del Pino M and Dolbeault J 2002 Best constants for Gagliardo-Nirenberg inequalities and applications to nonlinear diffusions J. Math. Pure Appl. 81 847-75

[9] Del Pino M and Dolbeault J 2003 The optimal Euclidean $L^{p}$-Sobolev logarithmic inequality J. Funct. Anal. 197 151-61

[10] Cordero-Erausquin D, Nazaret B and Villani C 2004 A mass-transportation approach to sharp Sobolev and Gagliardo-Nirenberg inequalities Adv. Math. 182 307-32

[11] Agueh M 2008 Sharp Gagliardo-Nirenberg inequalities via p-Laplacian type equations Nonlinear Differ. Eqns. Appl. NoDEA 15 457-72

[12] Pennini F, Plastino A and Ferri G L 2007 Semiclassical information from deformed and escort information measures Physica A 383 782-96

[13] Furuichi S 2009 On the maximum entropy principle and the minimization of the Fisher information in Tsallis statistics J. Math. Phys. 50013303

[14] Furuichi S 2010 On generalized Fisher information and Cramér-Rao type inequalities J. Phys.: Conf. Ser. 201012016

[15] Naudts J 2008 Generalised exponential families and associated entropy functions Entropy 10 131-49

[16] Naudts J 2009 The $q$-exponential family in statistical physics Central Eur. J. Phys. 7 405-13

[17] Lutwak E, Yang D and Zhang G 2005 Cramér-Rao and moment-entropy inequalities for Rényi entropy and generalized Fisher information IEEE Trans. Inform. Theory 51 473-8

[18] Lutwak E, Lv S, Yang D and Zhang G 2012 Extensions of Fisher information and Stam's inequality IEEE Trans. Inform. Theory 58 1319-27 
[19] Bercher J-F 2012 On generalized Cramér-Rao inequalities, generalized Fisher information and characterizations of generalized q-Gaussian distributions J. Phys. A: Math. Theor. 45255303

[20] Barankin E W 1949 Locally best unbiased estimates Ann. Math. Stat. 20 477-501

[21] Vajda I $1973 \chi^{\alpha}$-divergence and generalized Fisher information Trans. 6th Prague Conf. on Information Theory, Statistical Decision Functions and Random Processes pp 873-86

[22] Bercher J-F 2012 On a $(\beta, q)$-generalized Fisher information and inequalities involving $q$-Gaussian distributions J. Math. Phys. 53063303

[23] Stroock D W 1998 A Concise Introduction to the Theory of Integration (Berlin: Springer)

[24] Horn R A and Johnson C R 1990 Matrix Analysis (Cambridge: Cambridge University Press)

[25] Bonnans J F and Shapiro A 2000 Perturbation Analysis of Optimization Problems (Berlin: Springer)

[26] Limaye B V 1996 Functional Analysis (New Delhi: New Age International)

[27] Morrison T J 2000 Functional Analysis: An Introduction to Banach Space Theory 1st edn (New York: WileyInterscience)

[28] Lutwak E, Yang D and Zhang G 2007 Moment-entropy inequalities for a random vector IEEE Trans. Inform. Theory 53 1603-7

[29] Stam A J 1959 Some inequalities satisfied by the quantities of information of Fisher and Shannon Inf. Control 2 101-12

[30] Weyl H 1950 The Theory of Groups and Quantum Mechanics (New York: Dover)

[31] Folland GB and Sitaram A 1997 The uncertainty principle: a mathematical survey J. Fourier Anal. Appl. 3 207-38

[32] Angulo J C 1993 Uncertainty relationships in many-body systems J. Phys. A: Math. Gen. 266493

[33] Angulo J C 1994 Information entropy and uncertainty in d-dimensional many-body systems Phys. Rev. A 50 311-3

[34] Hirschman I I 1957 A note on entropy Am. J. Math. 79152

[35] Bialynicki-Birula I 2006 Formulation of the uncertainty relations in terms of the Rényi entropies Phys. Rev. A 74052101

[36] Zozor S and Vignat C 2007 On classes of non-Gaussian asymptotic minimizers in entropic uncertainty principles Physica A 375 499-517

[37] Sen K D (ed) 2011 Statistical Complexity: Applications in Electronic Structure 1st edn (Dordrecht: Springer)

[38] Dehesa J S, López-Rosa S and Manzano D 2011 Entropy and complexity analyses of $d$-dimensional quantum systems Statistical Complexity: Applications in Electronic Structure 1st edn ed K D Sen (Dordrecht: Springer) pp 129-66

[39] Zozor S, Portesi M, Sanchez-Moreno P and Dehesa J S 2011 Position-momentum uncertainty relations based on moments of arbitrary order Phys. Rev. A 83052107

[40] Beckner W 1975 Inequalities in Fourier analysis on $\mathbb{R}^{n}$ Proc. Natl Acad. Sci. USA 72 638-641 